\title{
Metastatic Disease Clinical Trial Setting
}

National Cancer Institute

\section{Source}

National Cancer Institute. Metastatic Disease Clinical Trial Setting. NCI Thesaurus. Code C124452.

A clinical trial or treatment setting in which the therapy of interest is used to treat metastatic disease. 